Check for updates

Cite this: Mater. Chem. Front., 2020, 4, 1315

Received 15th January 2020,

Accepted 14th February 2020

DOI: $10.1039 / d 0 q m 00026 d$

rsc.li/frontiers-materials

\section{Chemically modified nucleic acid biopolymers used in biosensing}

\author{
Lei Zhang, $\dagger^{a}$ Yanbing Yang, (D) $\dagger^{a}$ Jie Tan (D) *a and Quan Yuan (D) *ab
}

Biopolymers are highly designable and are often used in biosensing processes. As a biopolymer, a nucleic acid not only has excellent programmability and biocompatibility, but also has a certain molecular recognition function that can directly realize biological recognition in biosensors. However, the structural variety of natural nucleotides hinders the designability of nucleic acid biopolymers. The introduction of chemical modifications in nucleic acids can enrich the designability of nucleic acid biopolymers, thereby expanding their applications in biosensing. To date, there have been many reviews paying attention to biopolymers used in biosensing, but few reviews have focused on chemically modified nucleic acid biopolymers. Here, we review different kinds of assemblies based on chemically modified nucleic acid biopolymers. We summarize their advances in the field of biosensing. Furthermore, we present challenges and prospects in this field, aiming to provide a promising step for a versatile biosensor platform based on chemically modified nucleic acid biopolymers.

\section{Introduction}

Biosensing consists of a biorecognition process and a transduction process. ${ }^{1-4}$ Due to their high designability, biopolymers are often used to connect the biorecognition process with the transduction process. $^{5,6}$ It is worth noting that biopolymers can form

\footnotetext{
${ }^{a}$ Institute of Chemical Biology and Nanomedicine (ICBN), State Key Laboratory of Chemo/Biosensing and Chemometrics, College of Chemistry and Chemical Engineering, Hunan University, Changsha 410082, People's Republic of China. E-mail: tanjie0416@hnu.edu.cn

${ }^{b}$ Key Laboratory of Analytical Chemistry for Biology and Medicine (Ministry of Education), College of Chemistry and Molecular Sciences, Wuhan University, Wuhan 430072, People's Republic of China. E-mail: yuanquan@whu.edu.cn $\dagger$ These authors contributed equally.
}

nanostructures through a highly controllable dynamic assembly process, enabling the application of biosensing at the cellular or in vivo level. ${ }^{7-10}$

A nucleic acid, as a biopolymer, has excellent programmability and biocompatibility. ${ }^{11-16}$ In addition, nucleic acids with specific sequences can show a molecular recognition function and can be directly used in the biological recognition process. ${ }^{17,18}$

However, the structural variety of natural nucleotide hinders the designability of nucleic acid biopolymers, thereby limiting their applications in biosensing. ${ }^{12,19}$ The introduction of chemical modifications can enrich the functional groups of nucleic acids and enhance the intermolecular interactions between nucleic acids. ${ }^{20-22}$ Thus, chemically modified nucleic acids enable the design of nucleic acid biopolymers. ${ }^{23,24}$ Therefore, chemically

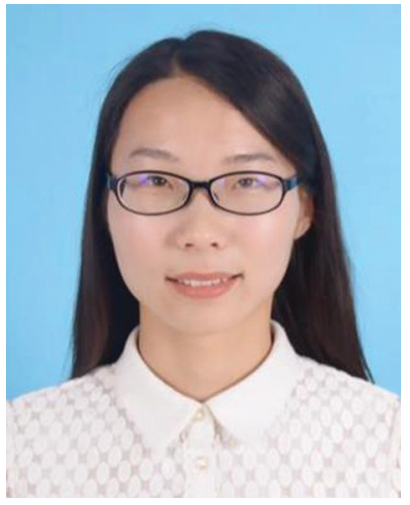

Lei Zhang
Lei Zhang obtained her BS degree from Henan Normal University. She is now pursuing her PhD degree in professor Quan Yuan's research group at the College of Chemistry and Chemical Engineering, Hunan University. Currently, her research interests are the design and applications of chemically modified nucleic acids.

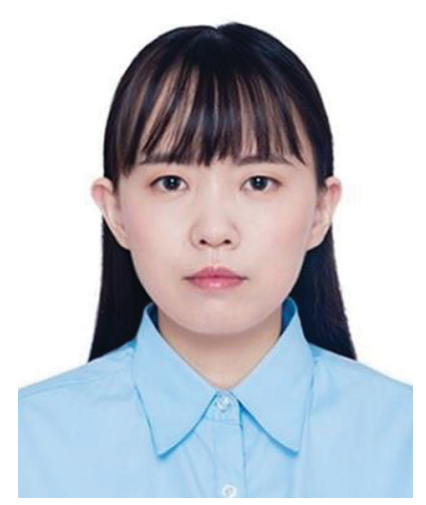

Yanbing Yang obtained her PhD degree from Wuhan University in 2017. Now, she is a postdoctoral fellow of Hunan University. Her research interests focus on the design of biosensors based on high-performance two-dimensional materials.

Yanbing Yang 
modified nucleic acid biopolymers hold great promise in the field of biosensing. ${ }^{25,26}$

To date, there have been many reviews on biopolymers used in biosensing, ${ }^{27-31}$ but few reviews have paid attention to chemically modified nucleic acid biopolymers. In this review, we focus on chemically modified nucleic acid biopolymers and their applications in biosensing. Specifically, we introduce the assembly methods of chemically modified nucleic acid biopolymers and the applications in biomolecule detection, controlled drug release, and gene therapy. Finally, the challenges and prospects in this field are also comprehensively discussed, aiming to provide a promising step for a versatile biosensor platform based on chemically modified nucleic acid biopolymers.

\section{Assembly of chemically modified nucleic acid biopolymers}

Nanoassemblies are often used in biosensing due to their similar size to biomolecules. ${ }^{32,33}$ Studies have shown that the design of nanostructures in two and three dimensions can greatly increase the sensitivity of biomolecule-targeted biosensors. ${ }^{34,35}$ Besides complementary base pairing interactions, chemical modifications can introduce new molecular interactions in nucleic acids. ${ }^{36}$ The new molecular forces enable the design of various geometric structures and bionic structures, creating opportunities for biosensing applications. ${ }^{21,37}$ In the following section, we mainly introduce assemblies based on chemically modified nucleic acid biopolymers with three assembly methods: complementary base pairing-mediated crosslinking method, hydrophilic-hydrophobic interaction-mediated crosslinking method, and metal ion-associated crosslinking method.

\subsection{Complementary base pairing-mediated crosslinking method}

It has been found that complementary base pairing is one of the major driving forces in DNA self-assembly processes. ${ }^{38-40}$ Previous research studies have shown that nucleic acids can be assembled into DNA nanostructures with controllable size and shape via complementary base pairing. ${ }^{41-45}$ The same goes for

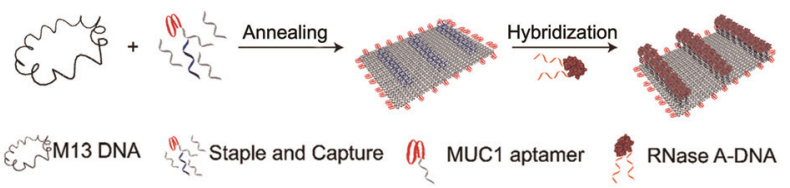

Fig. 1 Schematic diagram of two-dimensional nanosheet assembly. Reproduced with permission from ref. 47. Copyright 2019, American Chemical Society.

chemically modified nucleic acids, which could develop several nanostructures via base pairing interactions: nanosheets, hydrogels, tetrahedra, nanotubes, and nanoflowers.

DNA origami can be used as a carrier to load various components to expand its application. ${ }^{46}$ Very recently, Zhao et al. established a delivery platform based on DNA origami nanotechnology, which can transport payloads effectively for tumor treatment. ${ }^{47}$ They used a long M13mp 18 genomic DNA single strand as a scaffold, mixed with multiple predesigned staple strands and capture strands. These strands were annealed to fold into rectangular DNA origami nanosheets. The size of nanosheets is about $90 \mathrm{~nm} \times$ $60 \mathrm{~nm} \times 2 \mathrm{~nm}$. The complementary strands on the cytotoxic protein ribonuclease (RNase) A molecules were hybridized with capture strands to generate protein-DNA conjugates (Fig. 1). The nanosheets then transported RNase into the cells to exert the cytotoxicity function. To target cancer cells, the authors used aptamers as a guide. Aptamers are oligonucleotides with high affinity and selectivity for targets, which are obtained from in vitro screening. ${ }^{48}$ The aptamers targeting mucin 1 (MUC1) on a human breast adenocarcinoma cell line were integrated into the nanosheets, which significantly improved the effect of cancer treatment. Similarly, Chen et al. mixed an M13mp 18 single-strand DNA (ssDNA), tiles, and probes together to self-assemble into rectangular nanosheets. ${ }^{49}$ Subsequently, they used the nanosheets as a template, and precise assembly of proteases and corresponding coenzymes can be achieved. DNA origami nanostructures can precisely control the horizontal distance between enzymes so as to regulate the catalytic activity of the enzyme cascade. Zhou et al. used a similar method to prepare triangular nanosheets with a complete cavity, and the side length of the cavity was about $50 \mathrm{~nm} .{ }^{50}$ These studies show

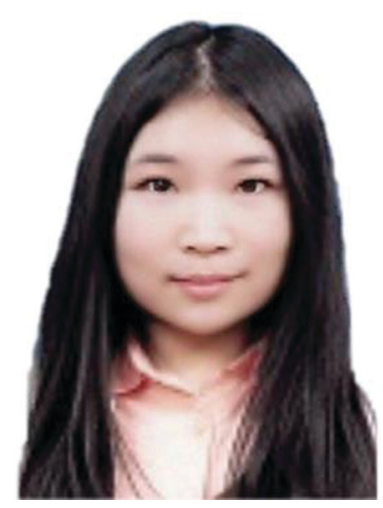

Jie Tan
Jie Tan earned her PhD degree from Zhejiang University in 2016. Then she joined the team of professor Weihong Tan at Hunan University as a postdoctoral fellow. Currently, she is an associate professor at Hunan University. Her main research interest is the design of chemically modified nucleic acids for biosensing.

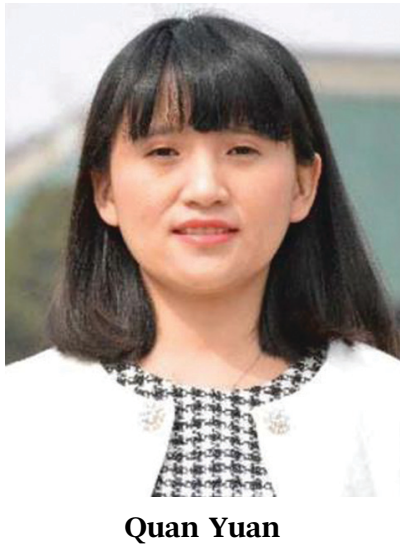

Quan Yuan received her BS degree from Wuhan University and $P h D$ degree from Peking University. Afterwards, she worked as a postdoctoral researcher in the group of professor Weihong Tan at the University of Florida. Currently, she is a professor at Hunan University. Her research interests are the construction of nucleic acid nanomaterials and related biomedical applications. 
that the framework design guided by DNA hybridization enables the fabrication of nanosheets of different shapes.

In addition to $2 \mathrm{D}$ origami nanosheets, it has been widely reported that a well-defined DNA hydrogel can be constructed through rational design and precise synthesis of DNA strands. ${ }^{51-53}$ As a case in point, rapid in situ formation of polypeptide-DNA hydrogels was reported by $\mathrm{Li}$ et $a .^{54}$ First, DNA-grafted polypeptides were prepared by an azide-alkyne click chemistry reaction. Five to six ssDNA motifs were conjugated to the backbone of each peptide, ensuring sufficient crosslinking sites. Additionally, double-stranded DNA served as a DNA linker containing two "sticky ends" that have the same sequence complementary to the DNA motifs. As a consequence of mixing DNA-grafted polypeptides and DNA linkers in a buffer solution, the fluidic solution changed to a transparent supramolecular crosslinking network within a few seconds (Fig. 2a). Tests showed that the mechanical strength of the chemically modified hydrogel was significantly enhanced. In another study, Li et al. also synthesized multifunctional supramolecular hydrogels by DNA-grafted polypeptides. ${ }^{55}$ The difference is that they used an " $\mathrm{X}$ "-shaped DNA as a crosslinker instead of the linear DNA linker. The " $X$ " shaped DNA strands are divided into two parts; one part is used as crosslinking sites, and the other part could be used for further functional modification. Subsequently, the two crosslinking sites are linked to the DNA-grafted polypeptide through precise base pairing to form a hydrogel structure (Fig. 2b). By this method, the resultant hydrogel can integrate various functional molecules to achieve applications in the biomedical field.

Three-dimensional DNA tetrahedral nanostructures have been widely studied because of their excellent mechanical stiffness and structural stability. ${ }^{56,57}$ Pei et al. did an elegant

a

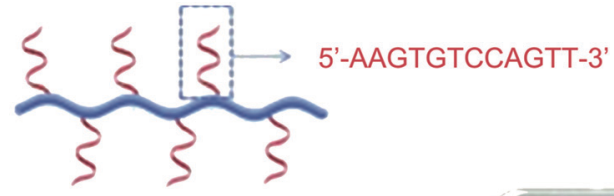

Polypeptide-DNA
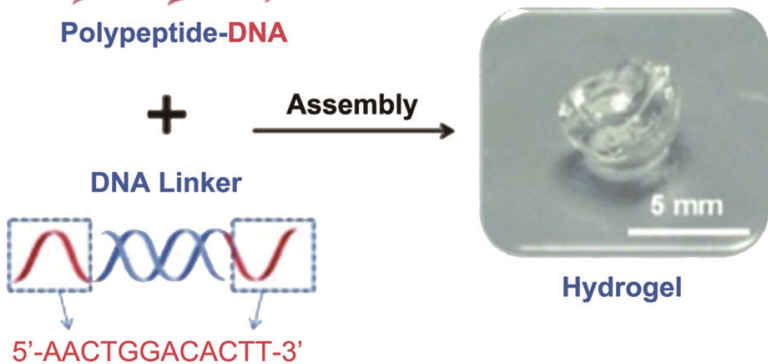

Hydrogel

5'-AACTGGACACTT-3'

b

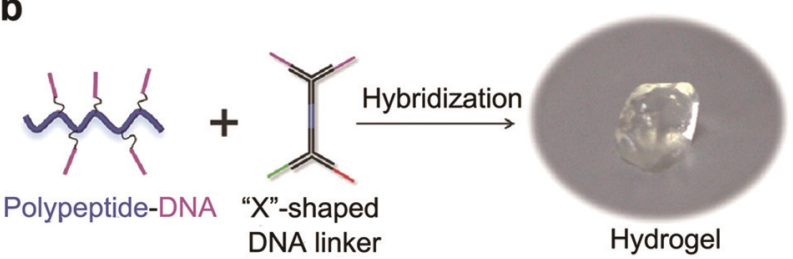

Fig. 2 Hydrogel assembly with linear (a) and X-shaped (b) DNA linkers Figures reproduced with permission from: a, ref. 54. Copyright 2015, Wiley-VCH; b, ref. 55. Copyright 2014, Wiley-VCH. work on the design of DNA tetrahedral nanostructures. They designed four ssDNAs with complementary sequences. ${ }^{58}$ Among them, three single chains are modified with the thiol group, and the other side chain contains a DNA probe. Four chains are mixed in an equivalent stoichiometric ratio, and the DNA tetrahedron is generated by annealing (heating, then rapid cooling) (Fig. 3a). This assembly process was fast (within two minutes) and the yield was high. This assembly method provides a new idea for constructing DNA tetrahedral probes with ordered orientation, controlled spacing and high stability. Besides, Li et al. assembled a DNA tetrahedron, which was constructed by three carboxyl group modified DNA strands (55 nucleotides) and a DNA strand containing an aptamer sequence (87 nucleotides). ${ }^{59}$ Four ssDNAs were quickly assembled into a tetrahedron with an edge length of $5.8 \mathrm{~nm}$ and each edge was an 18 base pair double helix (Fig. 3b). Polyacrylamide gel electrophoresis (PAGE) results showed that the DNA strands effectively self-assembled in the designed direction (Fig. 3c). The tetrahedral structure keeps the distance distribution of each chain the same, thereby reducing the spatial effect and improving the stability and recognition ability of biomolecules.

Apart from these structures, chemically modified nucleic acids can be assembled into tubular structures by complementary base pairing. According to the previous ssDNA tile assembly method, ${ }^{60}$ Kocabey et al. designed 24 functional oligonucleotides; after over 17 hours of annealing, the oligonucleotides were finally assembled into small tubular nanostructures with excellent stability (Fig. 4). ${ }^{61}$

In addition to the aforementioned DNA nanoassemblies, Zhang et al. synthesized submicron-scale DNA nanoflowers. ${ }^{62}$ A closed circular DNA containing the complementary sequences of aptamer Sgc8 was used as a template, and $\mathrm{Sgc} 8$ tandem repeat DNA sequences were generated by base pairing. The final long DNA sequence can spontaneously form nanoflower structures through crystallization, nucleation and growth processes. Finally, the authors introduced the DNA strands incorporating ferrocene $(\mathrm{Fc})$

a

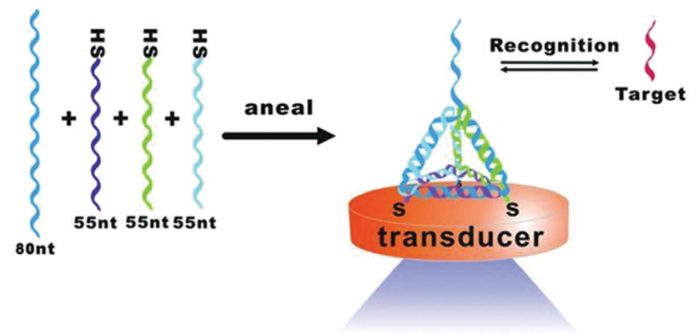

b

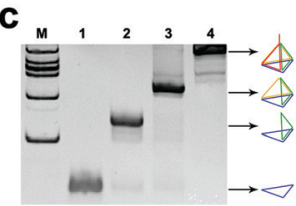

Fig. 3 DNA tetrahedra assembled by thiolated DNA strands (a) or carboxyl group modified DNA strands (b). (c) Analysis of DNA tetrahedra by PAGE. Figures reproduced with permission from: a, ref. 58. Copyright 2010, Wiley-VCH; b and c, ref. 59. Copyright 2015, American Chemical Society. 


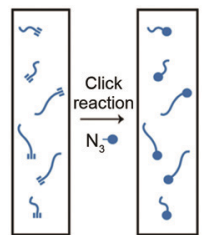

C8-Alkyne-du
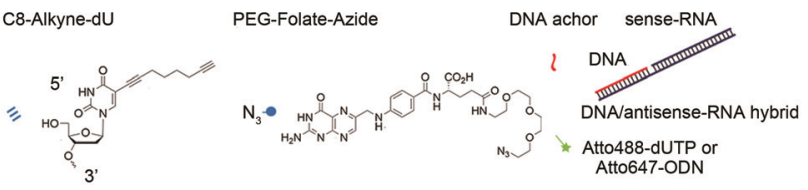

Fig. 4 Assembly of DNA nanotubes. Reproduced with permission from ref. 61. Copyright 2014, Molecular Diversity Preservation International.

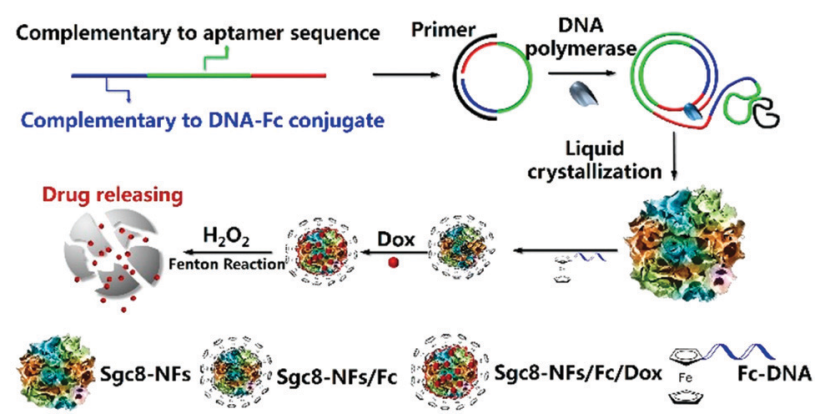

Fig. 5 Schematic diagram of nanoflower preparation. Reproduced with permission from ref. 62. Copyright 2019, American Chemical Society.

bases into the nanoflower (Fig. 5). The research indicated that the size of nanoflowers can be effectively adjusted by the introduction of Fc bases.

\subsection{Hydrophilic-hydrophobic interaction-mediated crosslinking method}

Similar to the assembly of amphiphilic polymers, the hydrophilic-hydrophobic interaction also plays a relevant role in the assembly processes of nucleic acid biopolymers. ${ }^{63}$ By modifying with hydrophobic groups, hydrophilic nucleic acids can turn into amphiphilic biopolymers, forming different structures through hydrophilic-hydrophobic interactions. ${ }^{64-67}$ Additionally, assemblies of specific size and shape can be obtained by changing the ratio of hydrophilic to hydrophobic chain length. ${ }^{65,68,69}$ In this section, we will introduce chemically modified nucleic acid assemblies formed via hydrophilic-hydrophobic interactions.

Linking hydrophobic units to hydrophilic units through covalent interactions is the main way to construct amphiphilic motifs. $^{70-72}$ Liu et al. connected hydrophilic DNA strands to hydrophobic diacyl lipids to form DNA-lipid molecules (Fig. 6a). ${ }^{73}$ They analysed the effect of DNA strand length on micelles. Atomic force microscopy (AFM) and dynamic light scattering (DLS) results showed that fine-tuning the length of the DNA sequence can achieve the purpose of adjusting the size of the micelles (Fig. $6 \mathrm{~b}$ and c). In a similar study, Wu et al. linked diacyl lipids to nucleic acid aptamers via polyethylene glycol to assemble a highly ordered micelle-like structure. ${ }^{74}$ In this structure, the nucleic acid strand
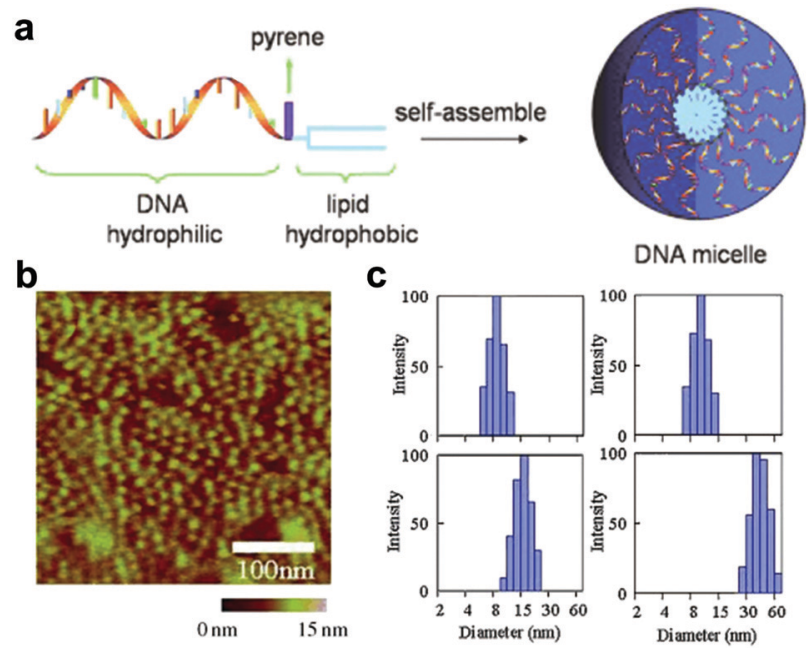

Fig. 6 (a) Illustration of assembly of diaycl-DNA micelles. AFM (b) and DLS (c) characterization of DNA micelles. Reproduced with permission from ref. 73. Copyright 2010, Wiley-VCH.

not only serves as the basis of the nanostructure but also performs a recognition function for a specific target. These aptamer-lipid assemblies enhance the binding ability to the target in a complex biological environment.

Inspired by the higher hydrophobicity, lower solubility, and stronger aggregation tendency of fluorocarbon groups, Zou et al. replaced the traditional diacyl chain with a fluorocarbon chain to construct micelles (Fig. 7). ${ }^{75}$ Micelles formed by chain amphiphilic polymers have higher stability, and the critical micelle concentration values are significantly reduced. Thus, creating new hydrophobic building units enables the formation of amphiphilic biomolecules with different properties and expands the versatility of nanomicelles.

Moreover, adding chemically modified nucleotides is another way to introduce hydrophobic groups into hydrophilic nucleic acid chains. ${ }^{76,77}$ Kimoto et al. used the unnatural base 7-(2-thienyl)imidazo[4,5-b]pyridine (Ds) as the hydrophobic moiety in the aptamer selection process. ${ }^{78}$ In their research, the addition of Ds bases not only improved the performance of aptamers, but also successfully introduced hydrophobicity into the DNA strands. Tan et al. integrated hydrophobic ferrocenes into hydrophilic DNA strands using a similar method and constructed aptamerferrocene assemblies (ApFAs) based on hydrophilic-hydrophobic interactions. ${ }^{68}$ Ferrocene bases are prepared by the reaction of $(S)$ 3amino-1,2-propanediol and ferrocene carboxylic acid. Then using

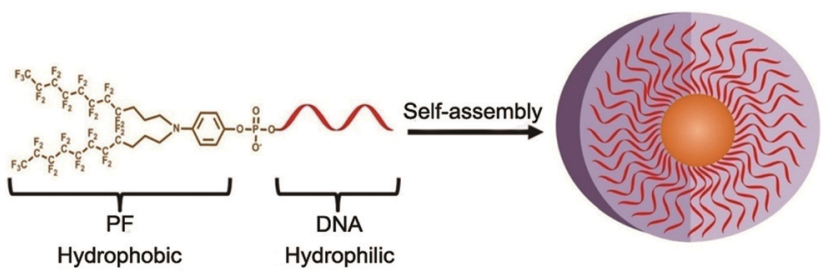

Fig. 7 Illustration of diperfluorodecyl-DNA Micelles. Reproduced with permission from ref. 75. Copyright 2018, American Chemical Society. 


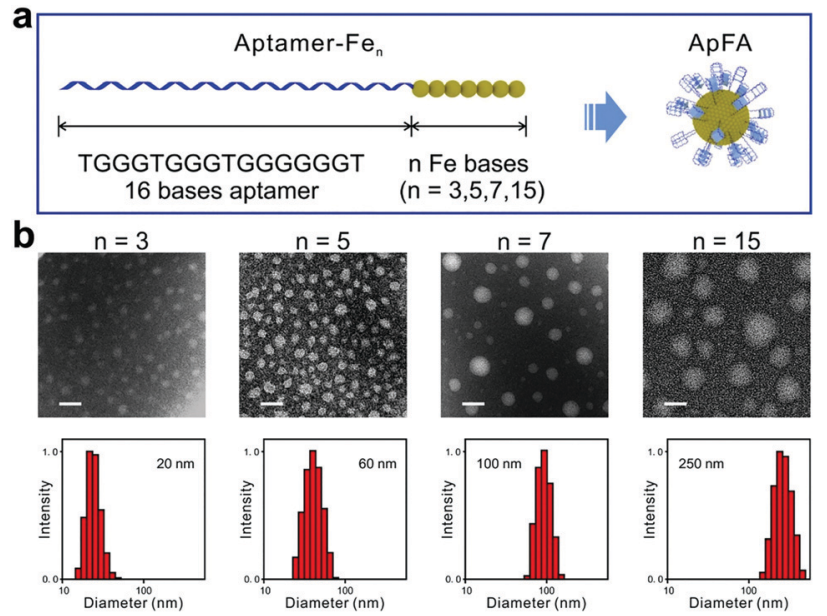

Fig. 8 (a) Schematic of ApFAs assembled by aptamers and different numbers of Fe nucleotides. (b) TEM and DLS characterization of the size of ApFAs. Reproduced with permission from ref. 68. Copyright 2019, Elsevier.

standard phosphoramidite chemistry, the chemically modified nucleic acids are synthesized on the DNA synthesizer by linking ferrocenes to natural bases. By designing synthetic procedures, the authors can synthesize oligonucleotides with specific bases at specific positions. The purified and desalted products can be assembled into micelles after being placed in the DPBS buffer for a certain time. Among them, the hydrophilic nucleic acid fragment is located on the surface of the assemblies in the form of a corona, and the hydrophobic ferrocene fragment forms the core (Fig. 8a). By regulating the number of hydrophobic ferrocene nucleotides, micelles of different sizes can be synthesized. Transmission electron microscopy (TEM) and DLS results showed that as the number of ferrocene nucleotide groups increased, the size of ApFAs increased gradually (Fig. 8b).

\subsection{Metal ion-associated crosslinking method}

Metal ions have aroused wide interest in biosensing since they play critical roles in biological organisms. ${ }^{79-82}$ Recently, nucleic acids have also been developed to form nanostructures with the assistance of metal ions. ${ }^{83,84}$

Lyu et al. designed a method for DNA micelle crosslinking in situ with the assistance of metal ions. ${ }^{85}$ The monomer that makes up the micelle is formed by the covalent attachment of a single stranded DNA and a hydrophobic diacyl lipid. Specifically, the monomer consists of three parts, the most important of which is the part that acts as a template chain (Fig. 9a). The template chain with specific sequences can adsorb different metal ions. The authors selected $\mathrm{Cu}^{2+}, \mathrm{Ag}^{+}$, and $\mathrm{Au}^{3+}$ to prepare different DNA micelles. Subsequently, the metal ions were reduced to zero-valent metals, which can be crosslinked into hollow or solid metal nuclei, thereby promoting the crosslinking of DNA micelles (Fig. 9b). In addition to the single-stranded micelles based on diacyl lipids, two cholesterol-modified double-stranded DNAs (dsDNAs) can also act as template domains. The authors have verified this. Compared with the single cholesterol-modified chain, the strong hydrophobic force produced by the two cholesterol-modified dsDNA a

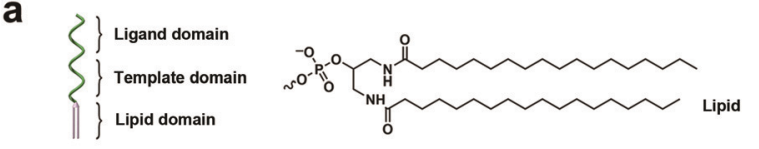

b

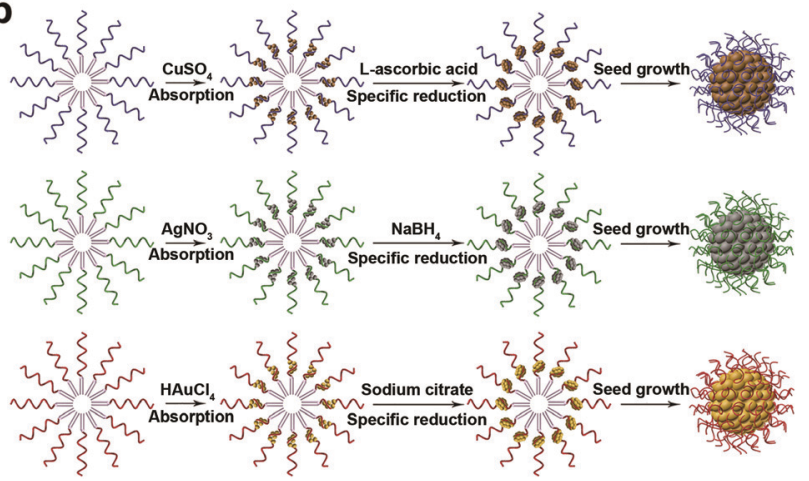

Fig. 9 (a) Monomer structure consisting of three domains. (b) Metal ionassisted crosslinking process of DNA micelles. Reproduced with permission from ref. 85. Copyright 2019, Elsevier.

can stabilize the micelle structure. Using $\mathrm{CuSO}_{4}$ as a copper source and $\mathrm{L}$-ascorbic acid as a reducing agent, it was found that cholesterolmodified dsDNA promoted micelle crosslinking, which can be demonstrated by fluorescence intensity and TEM characterization.

Inspired by the unique structures of the DNA G-quadruplexes, ${ }^{86}$ Lu et al. proposed that in the presence of $\mathrm{K}^{+}$ions, the crosslinking effect of G-quadruplexes was used to promote the formation of hydrogels. ${ }^{87}$ Acrydite-modified oligonucleotides are polymerized with acrylamide monomers to form copolymer chains under the catalysis of initiators. After adding $\mathrm{K}^{+}$ions, G-rich nucleic acid sequences self-assemble into G-quadruplex structures, so that polyacrylamide chains are cross-linked to form a DNA hydrogel (Fig. 10). To verify the role of $\mathrm{K}^{+}$in gel formation, the authors added crown ether that can capture $\mathrm{K}^{+}$ions, then the crosslinked G-quadruplex broke down, and the DNA hydrogel became a solution. Based on the fact that $\mathrm{K}^{+}$can stabilize the structure of G-quadruplex, Jin et al. designed a DNA sequence rich in G bases and developed a technique to stabilize the DNA micelle structure. ${ }^{88}$ With the addition of $\mathrm{K}^{+}$, adjacent guanine bases in the micellar canopy formed G-quadruplexes via Hoogsteen hydrogen bonding, and the hydrophobic lipid tails are confined within the micellar nucleus, thereby stabilizing the structure of

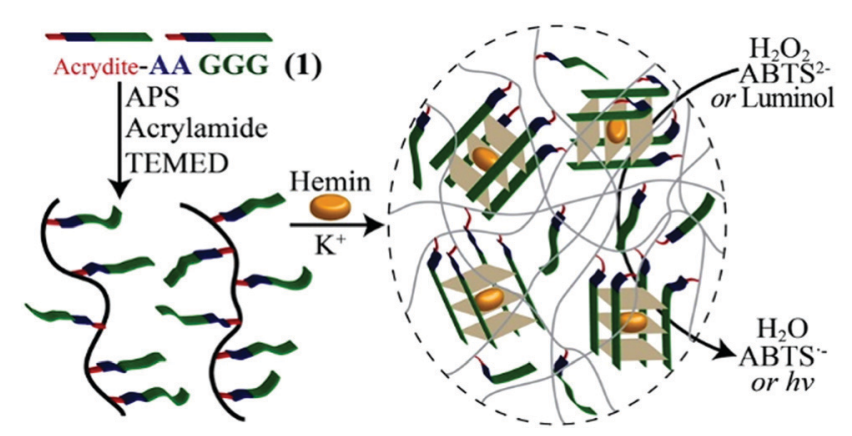

Fig. $10 \mathrm{~K}^{+}$-assisted crosslinking process of DNA hydrogels. Reproduced with permission from ref. 87. Copyright 2013, American Chemical Society. 


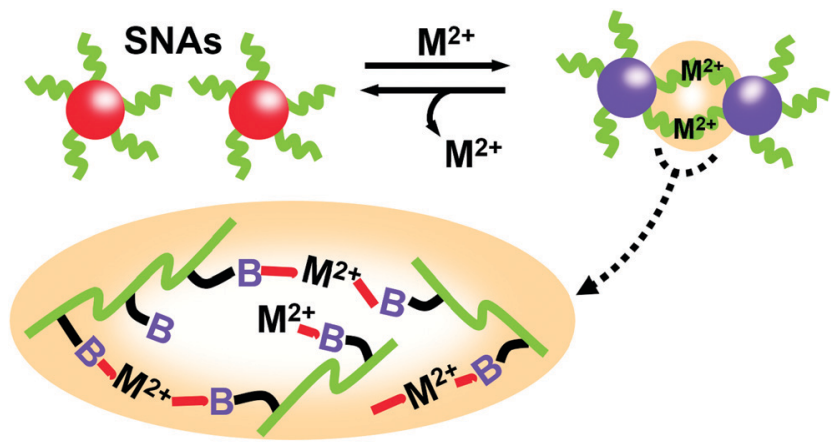

\section{B: Nucleobase (A, T, C, G) $B-M^{2+}$ : Coordinative Interactions}

Fig. 11 Schematic illustration of the $\mathrm{M}^{2+}$-assisted reversible assembly of SNAs.

micelles. These results showed that $\mathrm{K}^{+}$can promote the assembly of the gel and achieve reversible switching.

Unlike the works described above, the work by Joo et al. was based on the basic interaction between natural nucleobases and metal ions $\left(\mathrm{M}^{2+} \mathrm{s}\right)$ without any predesigned DNA sequence. ${ }^{89}$ In other words, even if the nucleic acid sequence of SNAs doesn't meet the requirements of complementary base pairing, it can induce the assembly of single-type spherical nucleic acids (SNAs). Taking $\mathrm{Cu}^{2+}$ as an example, a spherical nucleic acid solution with gold nanoparticles as the core was reacted with different concentrations of $\mathrm{Cu}^{2+}$ for $12 \mathrm{~h}$. As the concentration of $\mathrm{Cu}^{2+}$ increases, SNAs form larger aggregates. Furthermore, as the crosslinking reaction occurs, changes in the color of the solution can be observed with the naked eye, indicating the formation of assemblies. This research has shown that $\mathrm{M}^{2+} \mathrm{S}$ can coordinate with multiple bases of SNAs through coordinative interactions, thereby crosslinking into large aggregates (Fig. 11). Once $\mathrm{M}^{2+} \mathrm{s}$ are chelated by a stronger ligand, the assembled SNAs can be dissociated immediately. These studies showed that metal ions can mediate the formation of assemblies and can be reversibly reduced by removing metal ions.

\section{Application in biosensing}

Recently, much work has integrated biological functions into biosensing to achieve biomedical functions with high sensitivity and specificity. ${ }^{90-92}$ This requires that the materials used in biosensing can interact with and respond to target signals uniquely. Nucleic acid biopolymers show recognition ability to target molecules with good selectivity. ${ }^{93}$ The addition of chemical modifications can impart new chemical properties and biological functions to nucleic acid biopolymers. ${ }^{94,95}$ Therefore, the chemically modified nucleic acid biopolymers hold great potential to realize multifunctional biosensing. In this section, we will introduce the applications of chemically modified nucleic acid biopolymers in three parts: biomolecule detection, controlled drug release, and gene therapy.

\subsection{Detection of biomolecules}

The detection of biomolecules can provide us with a lot of information, which will help us make more accurate judgments on various life processes. ${ }^{96-98}$ Nucleic acids with specific sequences have the potential to recognize targets. ${ }^{99}$ However, the biological environment is so complicated that it limits the use of nucleic acid biopolymers in target recognition. ${ }^{100}$ Chemically modified nucleic acid biopolymers enable the specific and sensitive detection of targets. Here, we highlight the application of chemically modified nucleic acid biopolymers in biomolecule detection.

As exosomes are one of the biomolecules for early diagnosis of cancer, it is of great significance to detect exosomes with high sensitivity. ${ }^{101-103}$ Wang et al. fixed a nanotetrahedron (NTH) on the gold electrode and designed an aptamer-based biosensor (aptasensor) for detecting hepatocyte exosomes (Fig. 12a). ${ }^{104}$ When the sensor is incubated with the exosome suspension, the aptamer on the NTH binds to the exosomes specifically. The binding of the aptamer and exosome reduces the electrode surface area, which causes changes in electrical signals. Accordingly, changes in the electrical signal reflect the concentration of exosomes. Compared with the single-chain aptamer functionalized aptasensor, the detection sensitivity of the NTH-assisted aptasensor is increased by about 100 times (Fig. 12b).

In another study, Wu et al. constructed switchable aptamer micelle flares (SAMFs) for the detection of ATP molecules. ${ }^{105}$ The SAMF hydrophobic diacyl lipid tail is linked to a hydrophilic switchable aptamer head (Fig. 13a). When there are no targets, the aptamer probe retains the loop-stem structure, causing the fluorescence of the fluorophore to be quenched by an adjacent quencher. After binding to the target, the conformation of the aptamer changes and the fluorescence signal is restored. Based on this principle, a series of different concentrations of ATP are used to measure the response of SAMFs. It is observed that as the ATP concentration increases, the fluorescence signal intensity increases firstly and then reaches saturation (Fig. 13b). The lack of the target ATP prevents the stem-loop structure from opening, resulting in a lower fluorescence signal intensity. When the ATP molecules are added at 75 seconds, the fluorescence signal shows a straight upward trend and the response speed is fast (Fig. 13c). This study demonstrated that SAMFs can respond to ATP expression sensitively and detect ATP molecules through changes in fluorescent signals.

The detection of mRNA is of great significance for early tumor screening. ${ }^{106}$ Existing methods for detecting mRNA in cells have many limitations; for example, the probe is susceptible to interference by complex cellular environments, or the stability of the probe is poor. ${ }^{103}$ He et al. constructed a DNA tetrahedron

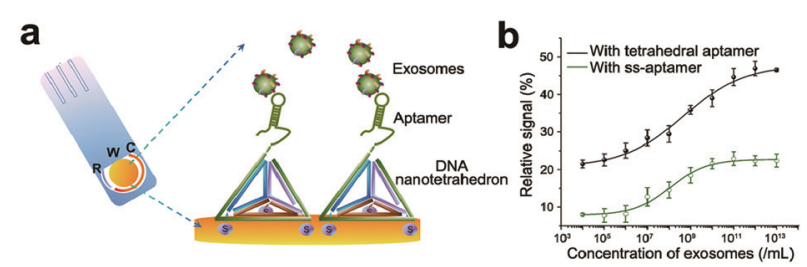

Fig. 12 (a) Illustration of the NTH-assisted aptasensor. (b) Comparison of signals between single-chain aptamer-assisted and NTH-assisted sensors. Reproduced with permission from ref. 104. Copyright 2017, American Chemical Society. 


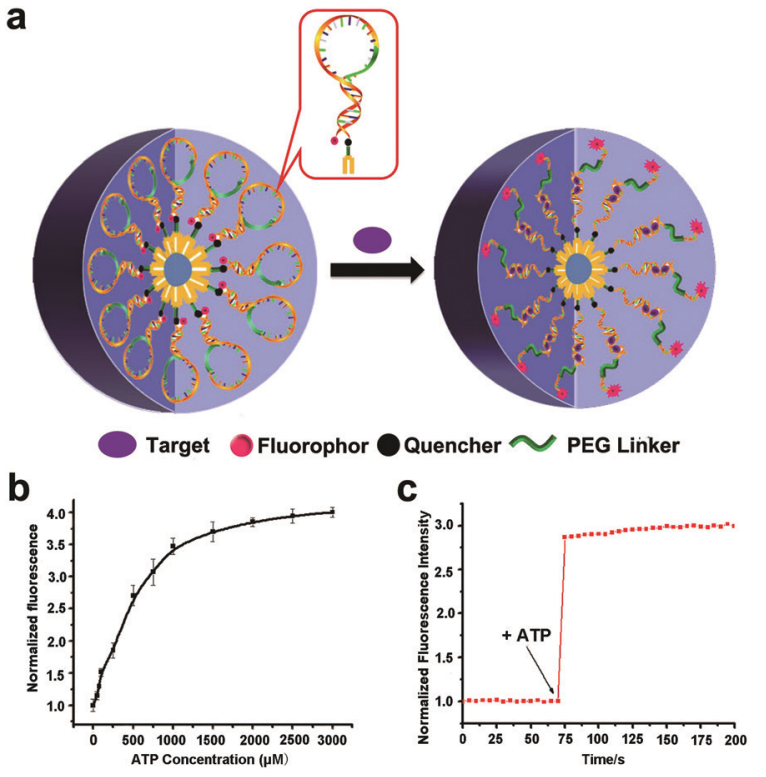

Fig. 13 (a) Structure of SAMFs and their working principle. (b) Relationship between the ATP concentration and fluorescent signal. (c) Effect of adding target molecules on fluorescence intensity. Reproduced with permission from ref. 105. Copyright 2013, American Chemical Society.

nanotweezer (DTNT) to solve the above problems. ${ }^{107}$ To be specific, the DTNT completed the mRNA detection with high sensitivity and high accuracy via the principle of fluorescence resonance energy transfer. They customized four DNA strands, two of which modified the Cy3 or Cy5 fluorophore respectively. In the absence of targets, the two fluorophores in the DTNT are far away, and the FRET is in the "off" state. When the target mRNA exists in the cell, the two fluorophores are located close to each other, which induces the generation of FRET and the signal changes from "off" to "on".

\subsection{Controlled drug release}

In recent years, the applications of smart drug delivery systems in the field of biosensing have been widely studied. ${ }^{108-111}$ Chemically modified nucleic acid biopolymers can also be used to construct smart drug delivery systems. ${ }^{112-114}$

A DNA origami structure is often used as a drug carrier system due to its excellent biocompatibility and spatially addressable functions. ${ }^{41,115} \mathrm{Jiang}$ et al. inserted anticancer drug doxorubicin (DOX) into DNA origami nanostructures through non-covalent bonding to reach a high level of drug loading efficiency. ${ }^{116}$ The DNA origami structure was effectively internalized into cells and remained in the tumor area for a long time. When the drug-loaded origami is exposed to low environmental $\mathrm{pH}$ or DNase, DNA nanostructures are slowly degraded to achieve controlled drug release (Fig. 14). Therefore, using DNA origami to carry drugs greatly improves the internalization ability of DOX, which will help the drug to exert its effect and kill tumor cells. In addition, the amphiphilic block copolymer can form a hydrophilic corona and a lipophilic core, and the core can be effectively loaded with hydrophobic anticancer drugs. Based on this, Alemdaroglu et al. assembled DNA-polypropylene oxide (PPO) into a spherical micelle structure. ${ }^{117}$ Dox is a hydrophobic drug, and due to the hydrophobic interaction

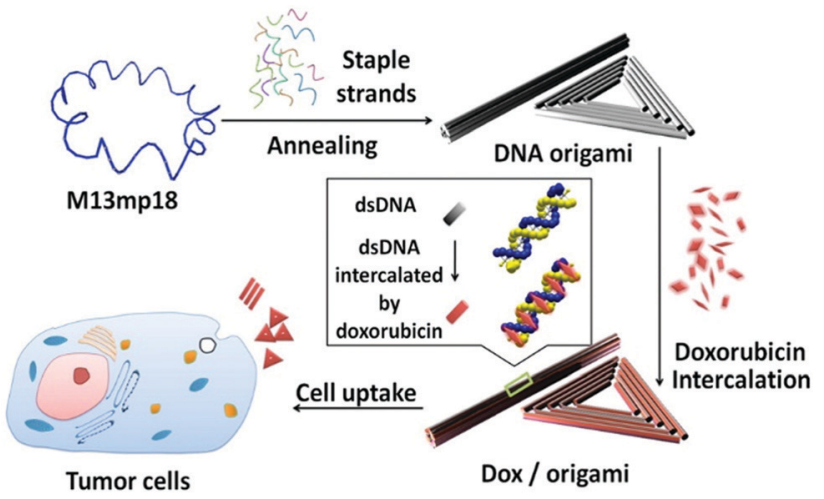

Fig. 14 DNA origami-based controlled drug release system. Reproduced with permission from ref. 116. Copyright 2012, American Chemical Society.

of Dox and PPO, drugs accumulate inside the micelles to form a drug delivery system.

Mitochondria play an important regulatory role in tumor growth. Jiang et al. developed a mitochondria-specific DNAtrain (MitoDNAtrs) drug delivery system using DNA as the basic unit (Fig. 15). ${ }^{118}$ The indocyanine dye Cy5.5 is used to provide the driving force, while the linear DNA strand is used as a carrier for transporting a good deal of antitumor drugs. Guided by Cy5.5, drugs are targeted to the mitochondrial region. The release of the drugs can be detected because Cy5.5 has a visualizable fluorescent signal. Applying MitoDNAtrs to breast cancer cells, and comparing the intracellular fluorescence signals at different times, it is clear that the intracellular fluorescence signals gradually increase. The results mean that drugs are gradually released from MitoDNAtrs, and the imaging method can be used to visualize the drug release process in time. Additionally, Li et al. also designed a controlled drug release system. ${ }^{119}$ They coupled a nucleolin-targeting aptamer (NucA) with paclitaxel (PTX) through a linker to form a nucleolin aptamer-paclitaxel conjugate (NucA-PTX). NucA directs PTX to ovarian tumor cells for action with little effect on normal cells. Once NucA-PTX enters the tumor cells through endocytosis, the lysosomal protease in the cell will cleave the linker in NucA-PTX, thereby achieving the controlled release of PTX.

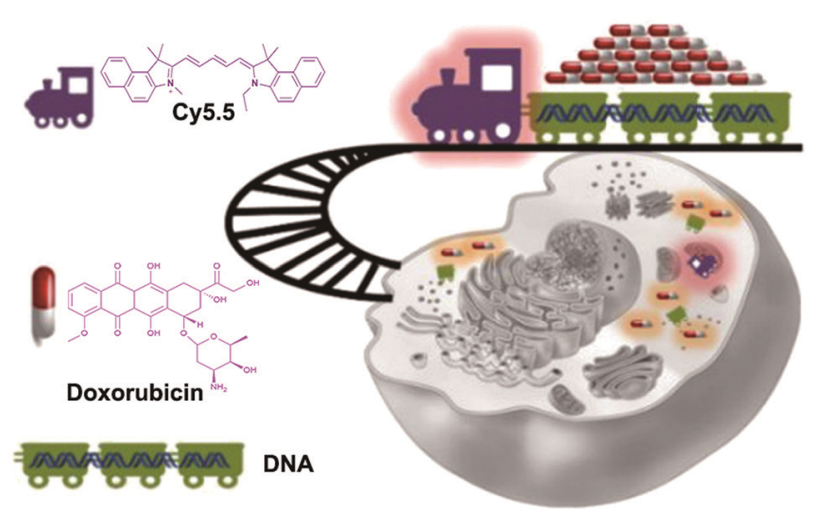

Fig. 15 Schematic of the MitoDNAtrs delivery system. Reproduced with permission from ref. 118. Copyright 2019, American Chemical Society. 
Interestingly, a high payload delivery system means a powerful lethality to tumor cells. ${ }^{120}$ As a typical example, Deng et al. proposed a general strategy to construct Aptamer-PolyproDrug Conjugates (ApPDCs); this method involved the assembly of aptamers targeting cancer cells, prodrugs and a biocompatible brush skeleton. ${ }^{121}$ Compared to traditional drug loading systems, ApPDCs have a high drug-to-aptamer ratio (DApR $\geq 40$ ). After the ApPDCs enter the cell, the reducing environment within the cell can trigger the efficient release of the prodrugs. By using prodrugs with different mechanisms, multi-drug synergy treatment can be achieved, and problems such as multi-drug resistance may be resolved.

Different from the above work, based on molecular engineering technology, the ratio of drugs can be adjusted accurately and multiple drugs can be precisely delivered to tumor cells. In this regard, Zhou et al. coupled circular bivalent aptamers with different drugs to prepare circular bivalent aptamer-drug conjugates (cb-ApDCs) with different drug dosing ratios. ${ }^{122}$ Drug release of cb-ApDCs can be monitored by confocal laser scanning microscopy; the ester bonds in cb-ApDCs can be cleaved by the esterase present in the late endosomes and lysosomes to realize the controlled release of drugs (Fig. 16b).
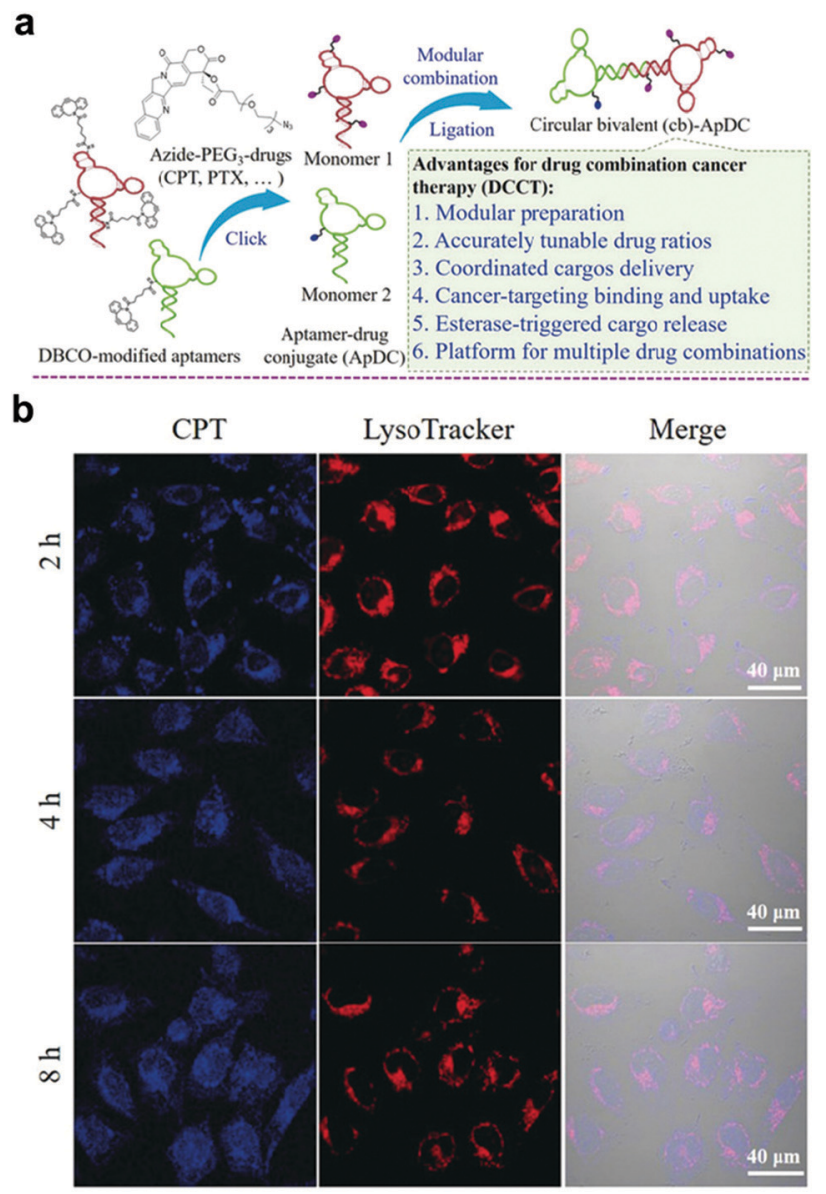

Fig. 16 (a) Preparation of cb-ApDCs. (b) Intracellular CPT release was monitored from intracellular cb-ApDCs. Reproduced with permission from ref. 122. Copyright 2019, Wiley-VCH.

\subsection{Gene therapy}

Chemically modified nucleic acid biopolymers can be combined with gene therapy technology to achieve highly efficient tumor treatment. ${ }^{123}$

Gene therapy has the ability to combat many gene-related diseases and it is a promising cancer treatment strategy. ${ }^{124}$ Li et al. designed a stimulus-responsive hydrogel for gene therapy, which was cross-linked by a building unit, a blocking and targeting unit and a linking unit (Fig. 17). ${ }^{125}$ Different functional elements were integrated into the three building units, such as therapeutic genes that can induce apoptosis, DNA ribozymes that can inhibit cell migration, and aptamers that can target cancer cells. Glutathione in cells can effectively cleave the disulfide bonds in the building blocks to achieve dissociation of nanohydrogels and release of multiple therapeutic genes. Moreover, Chen et al. synthesized molecular beacon micelle flares (MBMFs), which were assembled by diacyl lipids with circular DNA probes. ${ }^{126}$ The premise of using MBMFs for gene therapy is to hybridize with mRNA first, and there are two main therapeutic mechanisms: one is to prevent translation from target mRNAs by providing translational blocking, and the other is to form a DNA/RNA

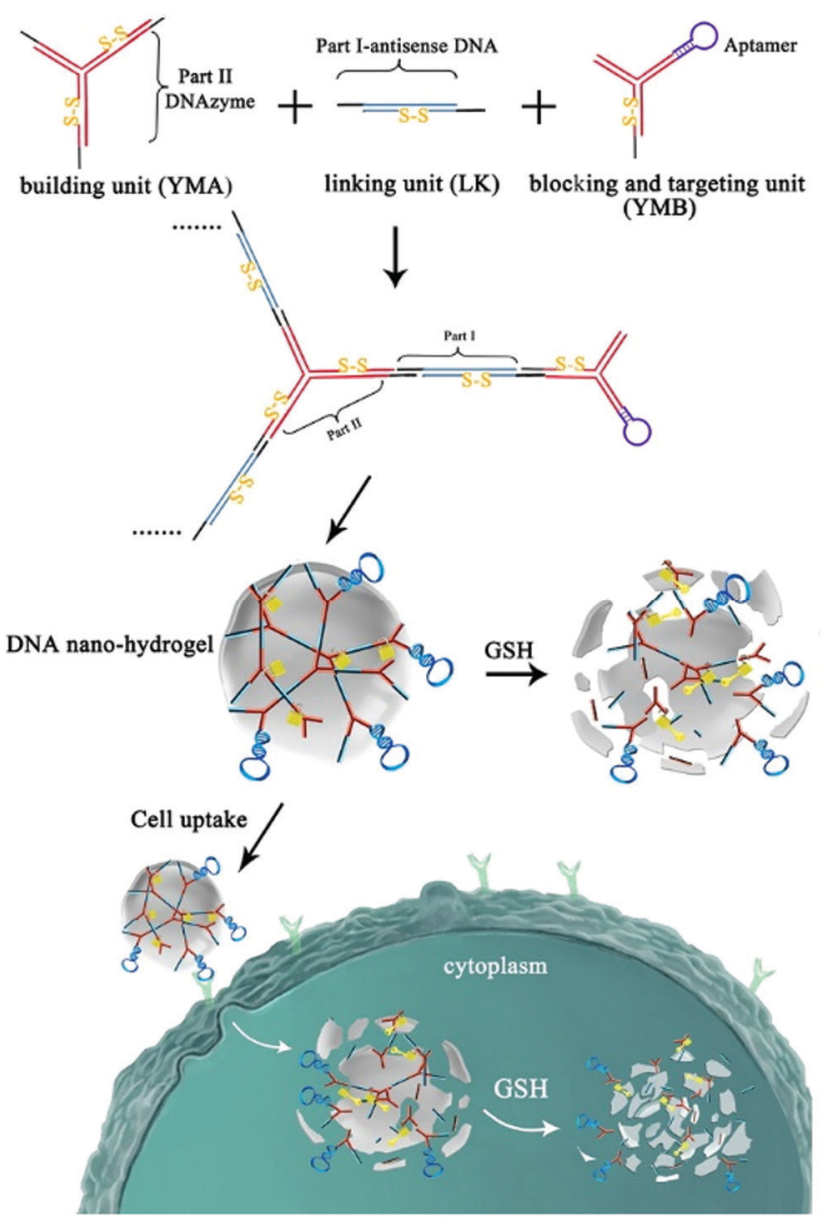

Fig. 17 Schematic of the hydrogel formation and gene therapy. Reproduced with permission from ref. 125. Copyright 2015, American Chemical Society. 


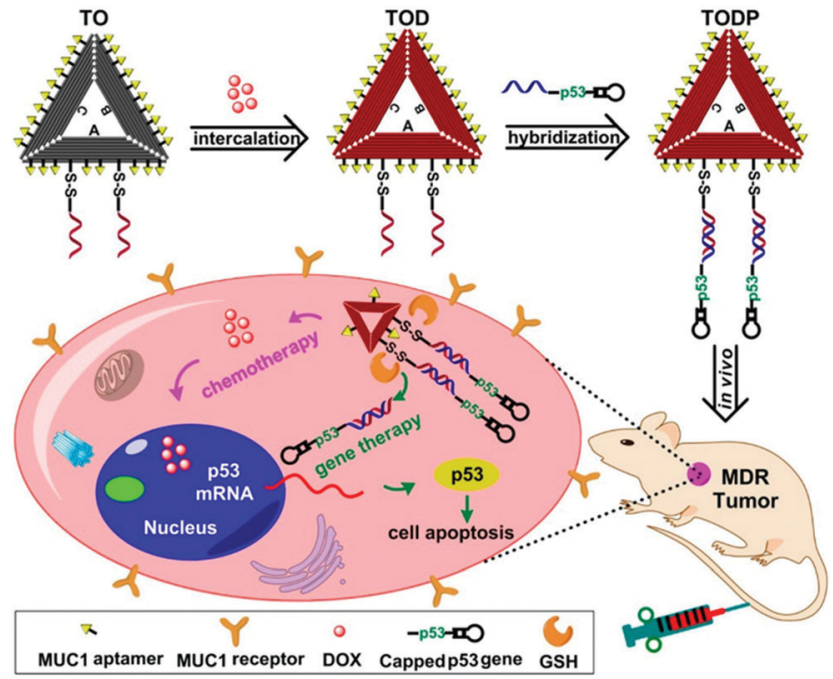

Fig. 18 Schematic of gene therapy based on a DNA origami structure. Reproduced with permission from ref. 127. Copyright 2018, American Chemical Society.

hybrid with the target mRNA, which is subsequently degraded by the RNase $\mathrm{H}$ enzyme. Using these two mechanisms can inhibit the growth of cancer cells and realize gene therapy. Liu $e t$ al. used gene therapy to enhance antitumor effects. ${ }^{127}$ Specifically, the authors constructed triangular DNA origami to load Dox, and then hybridized with the tumor therapeutic gene p53 to form a nano-kite assembly (Fig. 18). The assembly can effectively deliver p53 and DOX to tumor areas simultaneously, significantly enhancing the anti-tumor effect.

\section{Conclusion and outlook}

The addition of chemical modifications can impart rich structures and functions to nucleic acid biopolymers, attracting widespread attention from researchers. In this review, we introduced different cross-linking forms of chemically modified nucleic acid biopolymer nanostructures. Furthermore, we also summarized the applications of chemically modified nucleic acid biopolymers in biomolecule detection, controlled drug release, and gene therapy. These studies show the importance of chemically modified nucleic acid biopolymers in biosensing.

Although great achievements have been made, the application of chemically modified nucleic acid biopolymers in biosensing is still emerging and needs further improvement. For example, the efficiency of chemical modifications of nucleic acids needs to be further improved. Secondly, there is still an urgent requirement to develop nucleic acid biopolymers modified with different functional groups to achieve multifunctional biosensing. Finally, there are few chemically modified nucleic acid biopolymers available for clinical biosensing, so developing practical and safe chemically modified nucleic acid biopolymers is another key point. With the interdisciplinary development, chemically modified nucleic acid biopolymers have the potential to achieve greater promotions and breakthroughs in biosensing.

\section{Conflicts of interest}

There are no conflicts to declare.

\section{Acknowledgements}

This work was supported by the National Natural Science Foundation of China (21925401 and 21904037).

\section{Notes and references}

1 B. A. Cornell, V. Braach-Maksvytis, L. King, P. Osman, B. Raguse, L. Wieczorek and R. J. N. Pace, A biosensor that uses ion-channel switches, Nature, 1997, 387, 580-583.

2 A. J. Haes and R. P. Van Duyne, A Nanoscale Optical Biosensor: Sensitivity and Selectivity of an Approach Based on the Localized Surface Plasmon Resonance Spectroscopy of Triangular Silver Nanoparticles, J. Am. Chem. Soc., 2002, 124, 10596-10604.

3 M. A. Morales and J. M. Halpern, Guide to Selecting a Biorecognition Element for Biosensors, Bioconjugate Chem., 2018, 29, 3231-3239.

4 L. Soleymani and F. Li, Mechanistic Challenges and Advantages of Biosensor Miniaturization into the Nanoscale, ACS Sens., 2017, 2, 458-467.

5 J. Sun and R. N. Zuckermann, Peptoid Polymers: A Highly Designable Bioinspired Material, ACS Nano, 2013, 7, 4715-4732.

6 C. Rossner, Q. Tang, O. Glatter, M. Muller and P. Vana, Uniform Distance Scaling Behavior of Planet-Satellite Nanostructures Made by Star Polymers, Langmuir, 2017, 33, 2017-2026.

7 K. Doré, S. Dubus, H.-A. Ho, I. Lévesque, M. Brunette, G. Corbeil, M. Boissinot, G. Boivin, M. G. Bergeron, D. Boudreau and M. Leclerc, Fluorescent Polymeric Transducer for the Rapid, Simple, and Specific Detection of Nucleic Acids at the Zeptomole Level, J. Am. Chem. Soc., 2004, 126, 4240-4244.

8 Y. Zhao, S. Hu, H. Wang, K. Yu, Y. Guan, X. Liu, N. Li and F. Liu, DNA Dendrimer-Streptavidin Nanocomplex: an Efficient Signal Amplifier for Construction of Biosensing Platforms, Anal. Chem., 2017, 89, 6907-6914.

9 Y. Wang, L. P. Jiang, S. Zhou, S. Bi and J. J. Zhu, DNA Polymerase-Directed Hairpin Assembly for Targeted Drug Delivery and Amplified Biosensing, ACS Appl. Mater. Interfaces, 2016, 8, 26532-26540.

10 X. Fan, J. Y. Chung, Y. X. Lim, Z. Li and X. J. Loh, Review of Adaptive Programmable Materials and Their Bioapplications, ACS Appl. Mater. Interfaces, 2016, 8, 33351-33370.

11 M. Chandler and K. A. Afonin, Smart-Responsive Nucleic Acid Nanoparticles (NANPs) with the Potential to Modulate Immune Behavior, Nanomaterials, 2019, 9, 611.

12 S. Wan, L. Zhang, S. Wang, Y. Liu, C. Wu, C. Cui, H. Sun, M. Shi, Y. Jiang, L. Li, L. Qiu and W. Tan, Molecular Recognition-Based DNA Nanoassemblies on the Surfaces of Nanosized Exosomes, J. Am. Chem. Soc., 2017, 139, 5289-5292. 
13 S. Cai, J. Yan, H. Xiong, Y. Liu, D. Peng and Z. Liu, Investigations on the interface of nucleic acid aptamers and binding targets, Analyst, 2018, 143, 5317-5338.

14 C. Qi, S. Musetti, L. H. Fu, Y. J. Zhu and L. Huang, Biomolecule-assisted green synthesis of nanostructured calcium phosphates and their biomedical applications, Chem. Soc. Rev., 2019, 48, 2698-2737.

15 Y. J. Chen, B. Groves, R. A. Muscat and G. Seelig, DNA nanotechnology from the test tube to the cell, Nat. Nanotechnol., 2015, 10, 748-760.

16 C. S. Swenson, A. Velusamy, H. S. Argueta-Gonzalez and J. M. Heemstra, Bilingual Peptide Nucleic Acids: Encoding the Languages of Nucleic Acids and Proteins in a Single Self-Assembling Biopolymer, J. Am. Chem. Soc., 2019, 141, 19038-19047.

17 H. Wang, R. Yang, L. Yang and W. Tan, Nucleic Acid Conjugated Nanomaterials for Enhanced Molecular Recognition, ACS Nano, 2009, 3, 2451-2460.

18 L. Mo, J. Li, Q. Liu, L. Qiu and W. Tan, Nucleic acidfunctionalized transition metal nanosheets for biosensing applications, Biosens. Bioelectron., 2017, 89, 201-211.

19 N. Sugimoto, Designable DNA Functions toward New Nanobiotechnology, Bull. Chem. Soc. Jpn., 2009, 82, 1-10.

20 M. Xiao, W. Lai, T. Man, B. Chang, L. Li, A. R. Chandrasekaran and H. Pei, Rationally Engineered Nucleic Acid Architectures for Biosensing Applications, Chem. Rev., 2019, 119, 11631-11717.

21 D. Al Sulaiman, P. Cadinu, A. P. Ivanov, J. B. Edel and S. Ladame, Chemically Modified Hydrogel-Filled Nanopores: A Tunable Platform for Single-Molecule Sensing, Nano Lett., 2018, 18, 6084-6093.

22 M. Kuwahara and N. Sugimoto, Molecular Evolution of Functional Nucleic Acids with Chemical Modifications, Molecules, 2010, 15, 5423-5444.

23 K. A. Lennox and M. A. Behlke, Chemical modification and design of anti-miRNA oligonucleotides, Gene Ther., 2011, 18, 1111-1120.

24 P. A. Lichtor, Z. Chen, N. H. Elowe, J. C. Chen and D. R. Liu, Side chain determinants of biopolymer function during selection and replication, Nat. Chem. Biol., 2019, 15, 419-426.

25 I. K. Astakhova and J. Wengel, Scaffolding along Nucleic Acid Duplexes Using 2'-Amino-Locked Nucleic Acids, Acc. Chem. Res., 2014, 47, 1768-1777.

26 K. Holz, E. Schaudy, J. Lietard and M. M. Somoza, Multilevel patterning nucleic acid photolithography, Nat. Commun., 2019, 10, 3805.

27 L. Uzun and A. P. Turner, Molecularly-imprinted polymer sensors: realising their potential, Biosens. Bioelectron., 2016, 76, 131-144.

28 C. Wu and D. T. Chiu, Highly Fluorescent Semiconducting Polymer Dots for Biology and Medicine, Angew. Chem., Int. Ed., 2013, 52, 3086-3109.

29 Y. Wu, W. Wei and S. Liu, Target-Triggered Polymerization for Biosensing, Acc. Chem. Res., 2012, 45, 1441-1450.

30 M. Liu, Z. Li, Y. Li, J. Chen and Q. Yuan, Self-assembled nanozyme complexes with enhanced cascade activity and high stability for colorimetric detection of glucose, Chin. Chem. Lett., 2019, 30, 1009-1012.

31 S. Cosnier and M. Holzinger, Electrosynthesized polymers for biosensing, Chem. Soc. Rev., 2011, 40, 2146-2156.

32 H. Tran, K. Ronaldson, N. A. Bailey, N. A. Lynd, K. L. Killops, G. Vunjak-Novakovic and L. M. Campos, Hierarchically Ordered Nanopatterns for Spatial Control of Biomolecules, ACS Nano, 2014, 8, 11846-11853.

33 C. Rodriguez-Quijada, M. Sanchez-Purra, H. de Puig and K. Hamad-Schifferli, Physical Properties of Biomolecules at the Nanomaterial Interface, J. Phys. Chem. B, 2018, 122, 2827-2840.

34 T. Ming, Y. Wang, J. Luo, J. Liu, S. Sun, Y. Xing, G. Xiao, H. Jin and X. Cai, Folding Paper-Based Aptasensor Platform Coated with Novel Nanoassemblies for Instant and Highly Sensitive Detection of $17 \beta$-Estradiol, ACS Sens., 2019, 4, 3186-3194.

35 N. M. Robertson, M. S. Hizir, M. Balcioglu, M. Rana, H. Yumak, O. Ecevit and M. V. Yigit, Monitoring the Multitask Mechanism of DNase I Activity Using Graphene Nanoassemblies, Bioconjugate Chem., 2015, 26, 735-745.

36 C. Jin, J. He, J. Zou, W. Xuan, T. Fu, R. Wang and W. Tan, Phosphorylated lipid-conjugated oligonucleotide selectively anchors on cell membranes with high alkaline phosphatase expression, Nat. Commun., 2019, 10, 2704.

37 R. J. Banga, B. Meckes, S. P. Narayan, A. J. Sprangers, S. T. Nguyen and C. A. Mirkin, Cross-Linked Micellar Spherical Nucleic Acids from Thermoresponsive Templates, J. Am. Chem. Soc., 2017, 139, 4278-4281.

38 P. Chidchob, T. G. Edwardson, C. J. Serpell and H. F. Sleiman, Synergy of Two Assembly Languages in DNA Nanostructures: Self-Assembly of Sequence-Defined Polymers on DNA Cages, J. Am. Chem. Soc., 2016, 138, 4416-4425.

39 F. A. Aldaye, A. L. Palmer and H. F. Sleiman, Assembling Materials with DNA as the Guide, Science, 2008, 321, 1795-1799.

40 N. C. Seeman, DNA in a material world, Nature, 2003, 421, 427-431.

41 J. Fu, M. Liu, Y. Liu, N. W. Woodbury and H. Yan, Interenzyme Substrate Diffusion for an Enzyme Cascade Organized on Spatially Addressable DNA Nanostructures, J. Am. Chem. Soc., 2012, 134, 5516-5519.

42 S. Pal, Z. Deng, B. Ding, H. Yan and Y. Liu, DNA-OrigamiDirected Self-Assembly of Discrete Silver-Nanoparticle Architectures, Angew. Chem., Int. Ed., 2010, 49, 2700-2704.

43 R. P. Goodman, I. A. T. Schaap, C. F. Tardin, C. M. Erben, R. M. Berry, C. F. Schmidt and A. J. Turberfield, Rapid Chiral Assembly of Rigid DNA Building Blocks for Molecular Nanofabrication, Science, 2005, 310, 1661-1665.

44 Y. Yang, M. Endo, K. Hidaka and H. Sugiyama, PhotoControllable DNA Origami Nanostructures Assembling into Predesigned Multiorientational Patterns, J. Am. Chem. Soc., 2012, 134, 20645-20653.

45 M. Lin, J. Wang, G. Zhou, J. Wang, N. Wu, J. Lu, J. Gao, X. Chen, J. Shi, X. Zuo and C. Fan, Programmable Engineering of a Biosensing Interface with Tetrahedral DNA Nanostructures 
for Ultrasensitive DNA Detection, Angew. Chem., Int. Ed., 2015, 54, 2151-2155.

46 P. W. Rothemund, Folding DNA to create nanoscale shapes and patterns, Nature, 2006, 440, 297-302.

47 S. Zhao, F. Duan, S. Liu, T. Wu, Y. Shang, R. Tian, J. Liu, Z. G. Wang, Q. Jiang and B. Ding, Efficient intracellular delivery of RNase A using DNA origami carriers, ACS Appl. Mater. Interfaces, 2019, 11, 11112-11118.

48 N. N. Quang, A. Miodek, A. Cibiel and F. Ducongé, Selection of Aptamers Against Whole Living Cells: From Cell-SELEX to Identification of Biomarkers, Methods Mol. Biol., 2017, 1575, 253-272.

49 Y. Chen, G. Ke, Y. Ma, Z. Zhu, M. Liu, Y. Liu, H. Yan and C. J. Yang, A Synthetic Light-Driven Substrate Channeling System for Precise Regulation of Enzyme Cascade Activity Based on DNA Origami, J. Am. Chem. Soc., 2018, 140, 8990-8996.

50 C. Zhou, Y. Zhang, Y. Dong, F. Wu, D. Wang, L. Xin and D. Liu, Precisely Controlled 2D Free-Floating Nanosheets of Amphiphilic Molecules through Frame-Guided Assembly, Adv. Mater., 2016, 28, 9819-9823.

51 Y. S. Zhang and A. Khademhosseini, Advances in engineering hydrogels, Science, 2017, 356, 6337.

52 J. Wu, P. Li, C. Dong, H. Jiang, X. Bin, X. Gao, M. Qin, W. Wang, C. Bin and Y. Cao, Rationally designed synthetic protein hydrogels with predictable mechanical properties, Nat. Commun., 2018, 9, 620.

53 N. Kong, Q. Peng and H. Li, Rationally Designed Dynamic Protein Hydrogels with Reversibly Tunable Mechanical Properties, Adv. Funct. Mater., 2014, 24, 7310-7317.

54 C. Li, A. Faulkner-Jones, A. R. Dun, J. Jin, P. Chen, Y. Xing, Z. Yang, Z. Li, W. Shu, D. Liu and R. R. Duncan, Rapid Formation of a Supramolecular Polypeptide-DNA Hydrogel for In Situ Three-Dimensional Multilayer Bioprinting, Angew. Chem., Int. Ed., 2015, 54, 3957-3961.

55 C. Li, P. Chen, Y. Shao, X. Zhou, Y. Wu, Z. Yang, Z. Li, T. Weil and D. Liu, A Writable Polypeptide-DNA Hydrogel with Rationally Designed Multi-modification Sites, Small, 2015, 11, 1138-1143.

56 D. Zeng, H. Zhang, D. Zhu, J. Li, L. San, Z. Wang, C. Wang, Y. Wang, L. Wang, X. Zuo and X. Mi, A novel ultrasensitive electrochemical DNA sensor based on double tetrahedral nanostructures, Bioelectronics, 2015, 71, 434-438.

57 R. P. Goodman, M. Heilemann, S. Doose, C. M. Erben, A. N. Kapanidis and A. J. Turberfield, Reconfigurable, braced, three-dimensional DNA nanostructures, Nat. Nanotechnol., 2008, 3, 93-96.

58 H. Pei, N. Lu, Y. Wen, S. Song, Y. Liu, H. Yan and C. Fan, A DNA Nanostructure-based Biomolecular Probe Carrier Platform for Electrochemical Biosensing, Adv. Mater., 2010, 22, 4754-4758.

59 J. Li, C. Y. Hong, S. X. Wu, H. Liang, L. P. Wang, G. Huang, X. Chen, H. H. Yang, D. Shangguan and W. Tan, Facile Phase Transfer and Surface Biofunctionalization of Hydrophobic Nanoparticles Using Janus DNA Tetrahedron Nanostructures, J. Am. Chem. Soc., 2015, 137, 11210-11213.
60 B. Wei, M. Dai and P. Yin, Complex shapes self-assembled from single-stranded DNA tiles, Nature, 2012, 485, 623-626.

61 S. Kocabey, H. Meinl, I. S. MacPherson, V. Cassinelli, A. Manetto, S. Rothenfusser, T. Liedl and F. S. Lichtenegger, Cellular Uptake of Tile-Assembled DNA Nanotubes, Nanomaterials, 2014, 5, 47-60.

62 L. Zhang, R. Abdullah, X. Hu, H. Bai, H. Fan, L. He, H. Liang, J. Zou, Y. Liu, Y. Sun, X. Zhang and W. Tan, Engineering of Bioinspired, Size-Controllable, SelfDegradable Cancer-Targeting DNA Nanoflowers via the Incorporation of an Artificial Sandwich Base, J. Am. Chem. Soc., 2019, 141, 4282-4290.

63 Y. Kopel and N. Giovambattista, Comparative Study of Water-Mediated Interactions between Hydrophilic and Hydrophobic Nanoscale Surfaces, J. Phys. Chem. B, 2019, 123, 10814-10824.

64 S. Gao, X. Zheng, B. Jiao and L. Wang, Post-SELEX optimization of aptamers, Anal. Bioanal. Chem., 2016, 408, 4567-4573.

65 S. K. Albert, X. Hu and S. J. Park, Dynamic Nanostructures from DNA-Coupled Molecules, Polymers, and Nanoparticles, Small, 2019, 15, e1900504, DOI: 10.1002/smll.201900504.

66 Y. Jia, P. Gao, Y. Zhuang, M. Miao, X. Lou and F. Xia, Facile Probe Design: Fluorescent Amphiphilic Nucleic Acid Probes without Quencher Providing Telomerase Activity Imaging Inside Living Cells, Anal. Chem., 2016, 88, 6621-6626.

67 F. E. Alemdaroglu and A. Herrmann, DNA meets synthetic polymers-highly versatile hybrid materials, Org. Biomol. Chem., 2007, 5, 1311-1320.

68 J. Tan, H. Li, X. Hu, R. Abdullah, S. Xie, L. Zhang, M. Zhao, Q. Luo, Y. Li, Z. Sun, Q. Yuan and W. Tan, Size-tunable assemblies based on ferrocene-containing DNA polymers for spatially uniform penetration, Chem, 2019, 5, 1775-1792.

69 D. Wang, Y. Zhou, X. Li, X. Qu, Y. Deng, Z. Wang, C. He, Y. Zou, Y. Jin and Y. Liu, Mechanisms of pH-Sensitivity and Cellular Internalization of PEOz- $b$-PLA Micelles with Varied Hydrophilic/Hydrophobic Ratios and Intracellular Trafficking Routes and Fate of the Copolymer, ACS Appl. Mater. Interfaces, 2017, 9, 6916-6930.

70 P. Wei, T. R. Cook, X. Yan, F. Huang and P. J. Stang, A discrete amphiphilic organoplatinum(II) metallacycle with tunable lower critical solution temperature behavior, J. Am. Chem. Soc., 2014, 136, 15497-15500.

71 C. Bao, Y. Yin and Q. Zhang, Synthesis and assembly of laccase-polymer giant amphiphiles by self-catalyzed CuAAC click chemistry, Biomacromolecules, 2018, 19, 1539-1551.

72 C. Yao, A. Yuan, Z. Wang, H. Lei, L. Zhang, L. Guo and $\mathrm{X}$. Dong, Amphiphilic two-dimensional graphitic carbon nitride nanosheets for visible-light-driven phase-boundary photocatalysis, J. Mater. Chem. A, 2019, 7, 13071-13079.

73 H. Liu, Z. Zhu, H. Kang, Y. Wu, K. Sefan and W. Tan, DNAbased micelles: synthesis, micellar properties and sizedependent cell permeability, Chem. - Eur. J., 2010, 16, 3791-3797.

74 Y. Wu, K. Sefah, H. Liu, R. Wang and W. Tan, DNA aptamermicelle as an efficient detection/delivery vehicle toward cancer cells, Proc. Natl. Acad. Sci. U. S. A., 2010, 107, 5-10. 
75 J. Zou, C. Jin, R. Wang, H. Kuai, L. Zhang, X. Zhang, J. Li, L. Qiu and W. Tan, Fluorinated DNA micelles: synthesis and properties, Anal. Chem., 2018, 90, 6843-6850.

76 M. Anaya, M. Kwak, A. J. Musser, K. Mullen and A. Herrmann, Tunable hydrophobicity in DNA micelles: design, synthesis, and characterization of a new family of DNA amphiphiles, Chem. - Eur. J., 2010, 16, 12852-12859.

77 Y. X. Wu and Y. J. Kwon, Aptamers: The "evolution" of SELEX, Methods, 2016, 106, 21-28.

78 M. Kimoto, R. Yamashige, K. Matsunaga, S. Yokoyama and I. Hirao, Generation of high-affinity DNA aptamers using an expanded genetic alphabet, Nat. Biotechnol., 2013, 31, 453-457.

79 L. Qiu, T. Zhang, J. Jiang, C. Wu, G. Zhu, M. You, X. Chen, L. Zhang, C. Cui, R. Yu and W. Tan, Cell Membrane-Anchored Biosensors for Real-Time Monitoring of the Cellular Microenvironment, J. Am. Chem. Soc., 2014, 136, 13090-13093.

80 K. P. Carter, A. M. Young and A. E. Palmer, Fluorescent Sensors for Measuring Metal Ions in Living Systems, Chem. Rev., 2014, 114, 4564-4601.

$81 \mathrm{X}$. Qian and Z. Xu, Fluorescence imaging of metal ions implicated in diseases, Chem. Soc. Rev., 2015, 44, 4487-4493.

82 Y. Li, H. Zhong, Y. Huang and R. Zhao, Recent Advances in AIEgens for Metal Ion Biosensing and Bioimaging, Molecules, 2019, 24, 4593.

83 Z.-L. Zhang, Y.-Y. Wu, K. Xi, J.-P. Sang and Z.-J. Tan, Divalent Ion-Mediated DNA-DNA Interactions: A Comparative Study of Triplex and Duplex, Biophys. J., 2017, 113, 517-528.

84 Y. Wu, D. Wang, I. Willner, Y. Tian and L. Jiang, Smart DNA Hydrogel Integrated Nanochannels with High Ion Flux and Adjustable Selective Ionic Transport, Angew. Chem., Int. Ed., 2018, 57, 7790-7794.

85 Y. Lyu, Y. Guo, R. Cai, R. Peng, C. Hong, X. Chen, W. Hou, X. Li, J. Tan, Y. Zou, X. Zhang, Q. Liu and W. Tan, Spherically Directed Synthesis and Enhanced Cellular Internalization of Metal-Crosslinked DNA Micelles, Chem, 2019, 5, 913-928.

86 G. Biffi, D. Tannahill, J. McCafferty and S. Balasubramanian, Quantitative visualization of DNA G-quadruplex structures in human cells, Nat. Chem., 2013, 5, 182-186.

87 C. H. Lu, X. J. Qi, R. Orbach, H. H. Yang, I. Mironi-Harpaz, D. Seliktar and I. Willner, Switchable Catalytic Acrylamide Hydrogels Cross-Linked by Hemin/G-Quadruplexes, Nano Lett., 2013, 13, 1298-1302.

88 C. Jin, X. Liu, H. Bai, R. Wang, J. Tan, X. Peng and W. Tan, Engineering Stability-Tunable DNA Micelles Using Photocontrollable Dissociation of an Intermolecular G-Quadruplex, ACS Nano, 2017, 11, 12087-12093.

89 J. H. Joo and J. S. Lee, Divalent metal ion-mediated assembly of spherical nucleic acids: the case study of $\mathrm{Cu}^{2+}$, Phys. Chem. Chem. Phys., 2015, 17, 30292-30299.

90 Z. Tian, L. Ding, K. Li, Y. Song, T. Dou, J. Hou, X. Tian, L. Feng, G. Ge and J. Cui, Rational Design of a LongWavelength Fluorescent Probe for Highly Selective Sensing of Carboxylesterase 1 in Living Systems, Anal. Chem., 2019, 91, 5638-5645.
91 Q. Lin, Z. Li and Q. Yuan, Recent advances in autofluorescence-free biosensing and bioimaging based on persistent luminescence nanoparticles, Chin. Chem. Lett., 2019, 30, 1547-1556.

92 X. Cao, S. C. Flagg, X. Li, N. Chennamsetty, G. Balakrishnan and T. K. Das, Quadrupole Dalton-Based Controlled Proteolysis Method for Characterization of Higher Order Protein Structure, Anal. Chem., 2019, 91, 5339-5345.

$93 \mathrm{~J}$. Zhang, T. Lan and Y. Lu, Molecular Engineering of Functional Nucleic Acid Nanomaterials toward In Vivo Applications, Adv. Healthcare Mater., 2019, 8, e1801158, DOI: $10.1002 /$ adhm.201801158.

94 Q. Liu, C. Jin, Y. Wang, X. Fang, X. Zhang, Z. Chen and W. Tan, Aptamer-conjugated nanomaterials for specific cancer cell recognition and targeted cancer therapy, NPG Asia Mater., 2014, 6, e95, DOI: 10.1038/am.2014.12.

95 W. Kotkowiak, J. Wengel, C. J. Scotton and A. Pasternak, Improved RE31 Analogues Containing Modified Nucleic Acid Monomers: Thermodynamic, Structural, and Biological Effects, J. Med. Chem., 2019, 62, 2499-2507.

96 H. Zhang, Y. Liu, K. Zhang, J. Ji, J. Liu and B. Liu, Single Molecule Fluorescent Colocalization of Split Aptamers for Ultrasensitive Detection of Biomolecules, Anal. Chem., 2018, 90, 9315-9321.

97 C. Zong, M. Xu, L. J. Xu, T. Wei, X. Ma, X. S. Zheng, R. Hu and B. Ren, Surface-Enhanced Raman Spectroscopy for Bioanalysis: Reliability and Challenges, Chem. Rev., 2018, 118, 4946-4980.

98 F. Ma, Y. Li, B. Tang and C.-Y. Zhang, Fluorescent Biosensors Based on Single-Molecule Counting, Acc. Chem. Res., 2016, 49, 1722-1730.

99 J. Liu and Y. Lu, Preparation of aptamer-linked gold nanoparticle purple aggregates for colorimetric sensing of analytes, Nat. Protoc., 2006, 1, 246-252.

100 A. Borrmann and J. C. M. van Hest, Bioorthogonal chemistry in living organisms, Chem. Sci., 2014, 5, 2123-2134.

101 S. A. Melo, L. B. Luecke, C. Kahlert, A. F. Fernandez, S. T. Gammon, J. Kaye, V. S. LeBleu, E. A. Mittendorf, J. Weitz, N. Rahbari, C. Reissfelder, C. Pilarsky, M. F. Fraga, D. Piwnica-Worms and R. Kalluri, Glypican1 identifies cancer exosomes and facilitates early detection of cancer, Nature, 2015, 523, 177-182.

102 R. Nedaeinia, M. Manian, M. H. Jazayeri, M. Ranjbar, R. Salehi, M. Sharifi, F. Mohaghegh, M. Goli, S. H. Jahednia, A. Avan and M. Ghayour-Mobarhan, Circulating exosomes and exosomal microRNAs as biomarkers in gastrointestinal cancer, Cancer Gene Ther., 2017, 24, 48-56.

103 Y. H. Soung, S. Ford, V. Zhang and J. Chung, Exosomes in Cancer Diagnostics, Cancers, 2017, 9, 8.

104 S. Wang, L. Zhang, S. Wan, S. Cansiz, C. Cui, Y. Liu, R. Cai, C. Hong, I. T. Teng, M. Shi, Y. Wu, Y. Dong and W. Tan, Aptasensor with Expanded Nucleotide Using DNA Nanotetrahedra for Electrochemical Detection of Cancerous Exosomes, ACS Nano, 2017, 11, 3943-3949.

105 C. Wu, T. Chen, D. Han, M. You, L. Peng, S. Cansiz, G. Zhu, C. Li, X. Xiong, E. Jimenez, C. J. Yang and W. Tan, 
Engineering of Switchable Aptamer Micelle Flares for Molecular Imaging in Living Cells, ACS Nano, 2013, 7, 5724-5731.

106 A. E. Prigodich, P. S. Randeria, W. E. Briley, N. J. Kim, W. L. Daniel, D. A. Giljohann and C. A. Mirkin, Multiplexed Nanoflares: mRNA Detection in Live Cells, Anal. Chem., 2012, 84, 2062-2066.

107 L. He, D. Q. Lu, H. Liang, S. Xie, C. Luo, M. Hu, L. Xu, $\mathrm{X}$. Zhang and W. Tan, Fluorescence Resonance Energy Transfer-Based DNA Tetrahedron Nanotweezer for Highly Reliable Detection of Tumor-Related mRNA in Living Cells, ACS Nano, 2017, 11, 4060-4066.

108 Z. Wang, Y. Ju, Z. Ali, H. Yin, F. Sheng, J. Lin, B. Wang and Y. Hou, Near-infrared light and tumor microenvironment dual responsive size-switchable nanocapsules for multimodal tumor theranostics, Nat. Commun., 2019, 10, 1-12.

109 C. Argyo, V. Weiss, C. Bräuchle and T. Bein, Multifunctional mesoporous silica nanoparticles as a universal platform for drug delivery, Chem. Mater., 2013, 26, 435-451.

110 J. Liu, G. Yang, W. Zhu, Z. Dong, Y. Yang, Y. Chao and Z. Liu, Light-controlled drug release from singlet-oxygen sensitive nanoscale coordination polymers enabling cancer combination therapy, Biomaterials, 2017, 146, 40-48.

111 Y. Dai, H. Bi, X. Deng, C. Li, F. He, P. A. Ma, P. Yang and J. Lin, $808 \mathrm{~nm}$ near-infrared light controlled dual-drug release and cancer therapy in vivo by upconversion mesoporous silica nanostructures, J. Mater. Chem. B, 2017, 5, 2086-2095.

112 C. Chen, J. Geng, F. Pu, X. Yang, J. Ren and X. Qu, Polyvalent Nucleic Acid/Mesoporous Silica Nanoparticle Conjugates: Dual Stimuli-Responsive Vehicles for Intracellular Drug Delivery, Angew. Chem., Int. Ed., 2011, 50, 882-886.

113 J. Li, C. Fan, H. Pei, J. Shi and Q. Huang, Smart Drug Delivery Nanocarriers with Self-Assembled DNA Nanostructures, Adv. Mater., 2013, 25, 4386-4396.

114 B. Shi, M. Zheng, W. Tao, R. Chung, D. Jin, D. Ghaffari and O. C. Farokhzad, Challenges in DNA Delivery and Recent Advances in Multifunctional Polymeric DNA Delivery Systems, Biomacromolecules, 2017, 18, 2231-2246.

115 F. Zhang, S. Jiang, S. Wu, Y. Li, C. Mao, Y. Liu and H. Yan, Complex wireframe DNA origami nanostructures with multi-arm junction vertices, Nat. Nanotechnol., 2015, 10, 779-784.

116 Q. Jiang, C. Song, J. Nangreave, X. Liu, L. Lin, D. Qiu, Z. G. Wang, G. Zou, X. Liang, H. Yan and B. Ding, DNA Origami as a Carrier for Circumvention of Drug Resistance, J. Am. Chem. Soc., 2012, 134, 13396-13403.
117 F. E. Alemdaroglu, N. C. Alemdaroglu, P. Langguth and A. Herrmann, DNA Block Copolymer Micelles - A Combinatorial Tool for Cancer Nanotechnology, Adv. Mater., 2008, 20, 899-902.

118 T. Jiang, L. Zhou, H. Liu, P. Zhang, G. Liu, P. Gong, C. Li, W. Tan, J. Chen and L. Cai, Monitorable MitochondriaTargeting DNAtrain for Image-Guided Synergistic Cancer Therapy, Anal. Chem., 2019, 91, 6996-7000.

119 F. Li, J. Lu, J. Liu, C. Liang, M. Wang, L. Wang, D. Li, H. Yao, Q. Zhang, J. Wen, Z. K. Zhang, J. Li, Q. Lv, X. He, B. Guo, D. Guan, Y. Yu, L. Dang, X. Wu, Y. Li, G. Chen, F. Jiang, S. Sun, B. T. Zhang, A. Lu and G. Zhang, A watersoluble nucleolin aptamer-paclitaxel conjugate for tumorspecific targeting in ovarian cancer, Nat. Commun., 2017, 8, 1390.

120 L. Pan, Q. He, J. Liu, Y. Chen, M. Ma, L. Zhang and J. Shi, Nuclear-Targeted Drug Delivery of TAT Peptide-Conjugated Monodisperse Mesoporous Silica Nanoparticles, J. Am. Chem. Soc., 2012, 134, 5722-5725.

121 Z. Deng, Q. Yang, Y. Peng, J. He, S. Xu, D. Wang, T. Peng, R. Wang, X. Q. Wang and W. Tan, Polymeric Engineering of Aptamer-Drug Conjugates for Targeted Cancer Therapy, Bioconjugate Chem., 2020, 31, 37-42.

122 F. Zhou, P. Wang, Y. Peng, P. Zhang, Q. Huang, W. Sun, N. He, T. Fu, Z. Zhao, X. Fang and W. Tan, Molecular Engineering-Based Aptamer-Drug Conjugates with Accurate Tunability of Drug Ratios for Drug Combination Targeted Cancer Therapy, Angew. Chem., Int. Ed., 2019, 58, 11661-11665.

123 O. Madkhali, G. Mekhail and S. D. Wettig, Modified gelatin nanoparticles for gene delivery, Int. J. Pharm., 2019, 554, 224-234.

124 W. L. Chew, Immunity to CRISPR Cas9 and Cas12a therapeutics, Wiley Interdiscip. Rev.: Syst. Biol. Med., 2018, 10, 1-23.

125 J. Li, C. Zheng, S. Cansiz, C. Wu, J. Xu, C. Cui, Y. Liu, W. Hou, Y. Wang, L. Zhang, I. T. Teng, H. H. Yang and W. Tan, Selfassembly of DNA Nanohydrogels with Controllable Size and Stimuli-Responsive Property for Targeted Gene Regulation Therapy, J. Am. Chem. Soc., 2015, 137, 1412-1415.

126 T. Chen, C. S. Wu, E. Jimenez, Z. Zhu, J. G. Dajac, M. You, D. Han, X. Zhang and W. Tan, DNA Micelle Flares for Intracellular mRNA Imaging and Gene Therapy, Angew. Chem., Int. Ed., 2013, 52, 2012-2016.

127 J. Liu, L. Song, S. Liu, Q. Jiang, Q. Liu, N. Li, Z. G. Wang and B. Ding, A DNA-Based Nanocarrier for Efficient Gene Delivery and Combined Cancer Therapy, Nano Lett., 2018, 18, 3328-3334. 